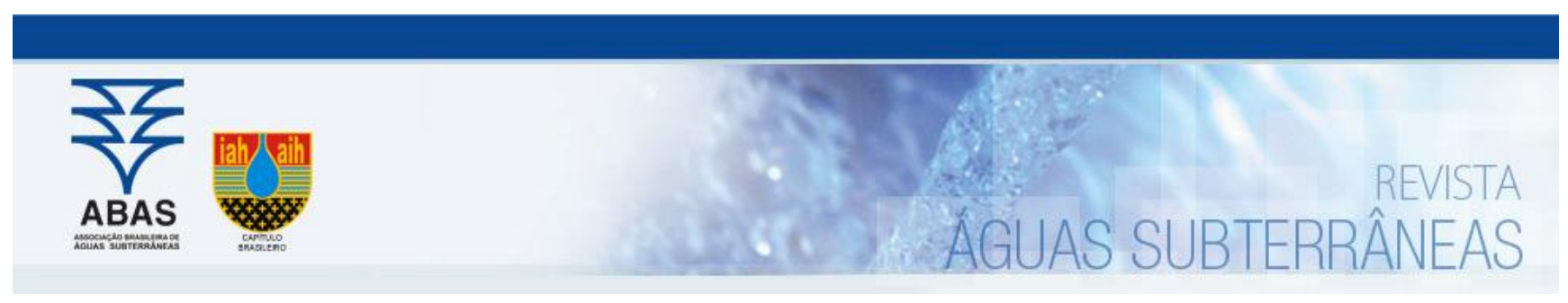

Artigos

\title{
Transmissividade e coeficiente de armazenamento obtidos a partir da difusividade em aquíferos confinados não drenantes
}

\section{Transmissivity and storage coefficient as obtained from diffusivity in non leaky confined aquifers}

\author{
Edilton Carneiro Feitosa ${ }^{\square}$ \\ 1 Universidade Federal de Pernambuco (UFPE), Recife, PE \\ ediltonf@yahoo.com.br
}

Palavras-chave:

Aquífero Cabeças.

Poço.

Difusividade.

Transmissividade.

Gurguéia/PI.

\section{Resumo}

Um conjunto de 18.000 valores teóricos de Difusividade (D) variando entre $0,012 \mathrm{~m}^{2} / \mathrm{s}$ e $8.914 \mathrm{~m}^{2} / \mathrm{s}$, em aquífero confinado, foi gerado aleatoriamente por um programa de computador, desenvolvido no âmbito do estudo, que utiliza um código para a solução da equação de Theis. Esse mesmo programa calculou 200 tempos de reação to de poços de observação posicionados a 15 distâncias diferentes do poço produtor, para 6 vazões diferentes, o que resultou na obtenção de 18.000 pares (to, D) obtidos aleatoriamente. A partir desses pares e de seus desdobramentos, e utilizando ajustes de leis potenciais e ajustes de leis logarítmicas, foi possível chegar à expressão analítica $D=\frac{e^{(2 \ln r-7,3)}}{t_{0}{ }_{0}^{0,93}}$ que fornece D em função da distância $r$ do poço produtor a um poço de observação $(m)$, e do tempo de reação to (min) observado neste último. Uma vez obtida a difusividade $D$, são apresentadas fórmulas que permitem desmembrá-la em seu dividendo $T=\frac{\mathrm{QW}(\mathrm{u})}{4 \pi \mathrm{s}^{\prime}}$ ( $\mathrm{s}^{\prime}$ = rebaixamento num dado tempo $\mathrm{t}^{\prime}$ em $\mathrm{min}$ ) e seu divisor $\mathrm{S}=\mathrm{T} / \mathrm{D}$, de forma unívoca. Os resultados obtidos para $\mathrm{D}$, T e S são validados por comparação com 40 valores desses parâmetros obtidos em 15 testes de aquífero realizados durante a década de 1980 no Aquífero Cabeças no Vale do Gurguéia, Piauí/Brasil. Esta abordagem é válida para a obtenção de valores confiáveis de $\mathrm{D}, \mathrm{T}$ e S, de modo a respaldar a elaboração adequada de projetos de poços e de baterias de poços.

Abstract

\section{Keywords:}

Cabeças Aquifer.

Well.

Diffusivity.

Transmissivity.

Vale do Gurguéia.

Revisado por pares.

Recebido em: 15/07/2019.

Aprovado em: 27/12/2019.
A specific code based on Theis equation was written which generated a random series of 18,000 theoretical Diffusivities (D) ranging from $0.012 \mathrm{~m}^{2} / \mathrm{s}$ to $8,914 \mathrm{~m}^{2} / \mathrm{s}$ in a non-leaky confined homogeneous aquifer. The code also served to provide 200 reaction times to (also called time lags or delay times) for observation wells placed at 15 different distances from the pumping well, for 6 different discharges, achieving eventually 18,000 random pairs (to, D). These pairs and its further developments were subjected to power regressions and logarithmic regressions which led to the analytical expression $D=\frac{e^{(2 \ln r-7,3)}}{t_{0} 0,93}$ for $D$ as a function of the distance $r(m)$ from the pumping well to an observation well and of the reaction time to $(\mathrm{min})$ of the later. Once $\mathrm{D}$ is known, further expressions are proposed which split it into its constituent's $\mathrm{T}=\frac{\mathrm{QW}(\mathrm{u})}{4 \pi \mathrm{s}^{\prime}}\left(\mathrm{S}^{\prime}=\right.$ drawdown at a time $\mathrm{t}^{\prime}$ in $\mathrm{min}$ ) and storativity $(\mathrm{S}=\mathrm{T} / \mathrm{D})$. The values obtained for $\mathrm{D}, \mathrm{T}$, and $\mathrm{S}$ are validated by comparison to 40 values of these parameters obtained from 15 long term aquifer tests performed during the 1980 s in the Cabeças Aquifer, Vale do Gurguéia/Piauí/Brazil. This approach is considered as suitable for providing reliable values of $\mathrm{D}, \mathrm{T}$, and $\mathrm{S}$ which are required for supporting design, construction, and installation of wells and well fields.

DOI: http:/dx.doi.org/10.14295/ras.v34i1.29580

\section{INTRODUÇÃO}

A caracterização do fluxo subterrâneo nos meios porosos exige o conhecimento de dois parâmetros hidrodinâmicos fundamentais: transmissividade hidráulica $(T)$ e coeficiente de armazenamento (S). Esses parâmetros são classicamente obtidos em testes de aquífero convencionais (FETTER, 1994). Em áreas já submetidas a uma explotação extensiva, entretanto, torna-se muito difícil a realização desses testes, sobretudo em face das superposições de escoamento decorrentes dos muitos e variados regimes de bombeamento sendo praticados. Essa dificuldade, muito frequente, levou à busca de uma abordagem alternativa para a obtenção dos referidos parâmetros hidrodinâmicos. O artigo constitui um esforço desenvolvido nesse sentido.

A equação diferencial geral que rege o fluxo subterrâneo em meio homogêneo e isotrópico é:

$$
\partial^{2} h / \partial x^{2}+\partial^{2} h / \partial y^{2}+\partial^{2} h / \partial z^{2}=S / T h / \partial t
$$

em que: 


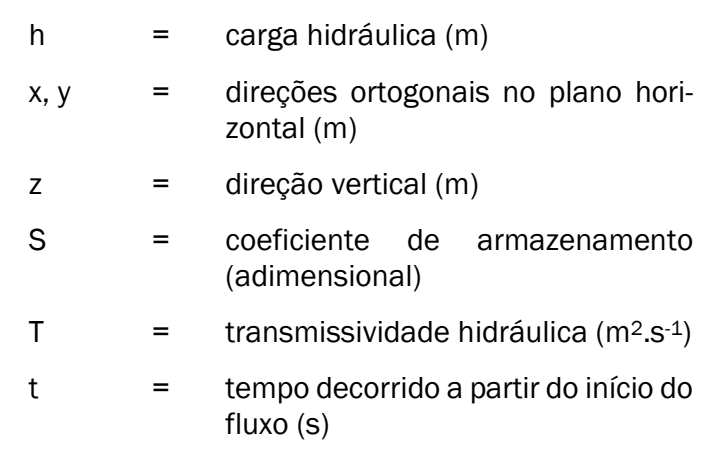

Soluções analíticas da equação (1) são disponíveis para as situações hidrogeológicas mais comuns, (i.e., aquíferos confinado, semiconfinado e livre). Para aquíferos confinados a solução da equação (1) para fluxo radial para um poço é a função analítica (2), denominada comumente de equação de Theis (1935).

$h_{0}-h=s_{w}=\frac{Q}{4 \pi T} W(u)$

em que:

$$
\begin{aligned}
& \text { ho = carga hidráulica inicial }(\mathrm{m}) \\
& \mathrm{h}=\text { carga hidráulica num dado tempo } \mathrm{t} \\
& \text { (m) } \\
& \mathrm{S}_{\mathrm{w}}=\text { rebaixamento num dado tempo } \mathrm{t} \\
& \text { (m) } \\
& \text { Q = vazão constante de bombeamento } \\
& \left(\mathrm{m}^{3} \cdot \mathrm{s}^{-1}\right) \\
& \mathrm{T}=\text { transmissividade hidráulica }\left(\mathrm{m}^{2} . \mathrm{s}^{-1}\right) \\
& \mathrm{W}(\mathrm{u})=\text { função de Theis dada por } \int_{u}^{\infty} \frac{-\mathrm{e}^{\mathrm{u}}}{\mathrm{u}} \mathrm{du} \text {. }
\end{aligned}
$$
$\mathrm{W}(\mathrm{u})$ pode ser aproximada pela série convergente infinita:

$$
W(u) \cong-0,5772-\ln u+u-\frac{u^{2}}{2.2 !}+\frac{u^{3}}{3.3 !}-\frac{u^{4}}{4.4 !} \ldots \ldots \ldots
$$

em que:

$$
\begin{array}{lll}
\mathrm{u} & = & \mathrm{r}^{2} \mathrm{~S} / 4 \mathrm{Tt} \quad \text { em que: } \\
\mathrm{r} & = & \begin{array}{l}
\text { distância do poço bombeado ao } \\
\text { ponto onde se deseja calcular o } \\
\text { rebaixamento } \mathrm{S}_{\mathrm{w}}(\mathrm{m}) .
\end{array} \\
\mathrm{S} & =\quad \begin{array}{l}
\text { coeficiente de armazenamento } \\
\text { (adimensional) }
\end{array} \\
\mathrm{t} & =\quad \begin{array}{l}
\text { tempo contado a partir do início } \\
\text { do bombeamento }(\mathrm{s}) .
\end{array}
\end{array}
$$

Para valores de $u$ muito pequenos $(u<0,01)$ os dois primeiros termos da série (3) aproximam satisfatoriamente o valor de $\mathrm{W}(\mathrm{u})$.

Tem-se, assim (COOPER; JACOB, 1946):

$W(u) \cong-0,5772-\ln u$

e

$h_{0}-h=s_{w}=\frac{Q}{4 \pi T}(-0,5772-\ln u)$

Desenvolvendo convenientemente a equação (5) acima se chega à chamada aproximação de Cooper e Jacob (equação 6) que é válida para pequenas distâncias do ponto de observação ao poço bombeado e longos tempos de bombeamento.
$S_{W}=\frac{Q}{4 \pi T} \ln \frac{2,25 T t}{r^{2} S}$

Custodio e Llamas (1983) manipulando a equação (6), para rebaixamento nulo $\left(s_{w}=0\right)$, chegaram a uma expressão que permite obter estimativas da difusividade $\mathrm{D}$ (equação 7 ).

$D=\frac{r^{2}}{2,25 \Delta t}$

em que:

$$
\begin{aligned}
& \mathrm{r}=\text { distância do poço bombeado a um } \\
& \text { poço de observação }(m) \text {. }
\end{aligned}
$$

Considerando, entretanto, que a equação (7) deriva da aproximação de Cooper e Jacob (equação 6), ela é também válida para pequenas distâncias do ponto de observação ao poço bombeado e longos tempos de bombeamento. Essa limitação restringe muito sua aplicação à avaliação de difusividades a partir de interferências entre poços.

O trabalho pretende discutir a obtenção de uma expressão baseada na equação de Theis, destinada a obter estimativas confiáveis de $\mathrm{D}$ a partir do tempo de reação (to) de poços de observação situados a qualquer distância do poço bombeado, bem como discute também uma abordagem que visa à obtenção de T e S, a partir de D.

A difusividade (D) é uma constante do meio poroso, definida como a razão $\mathrm{T} / \mathrm{S}\left(\mathrm{m}^{2} / \mathrm{s}\right)$. Seu papel na equação de Theis e, consequentemente, no comportamento real dos aquíferos confinados, depende essencialmente dos valores da transmissividade $\mathrm{T}$, com o coeficiente de armazenamento desempenhando papel pouco significativo. Para uma difusividade constante, os diferentes pares $(T, S)$, que satisfazem a razão $T / S$, definem diferentes curvas de rebaixamento. É por isso que os testes de aquífero fornecem de forma unívoca os parâmetros do meio.

\section{METODOLOGIA}

\subsection{Pesquisa Bibliográfica}

A maior parte das referências encontradas na literatura sobre avaliação da difusividade de aquíferos prioriza as suas relações com fenômenos externos a eles. Deve-se a Boussinesq (1877, apud Silva, 1984) o primeiro estudo sobre a propagação de ondas potenciométricas sinusoidais em resposta ao estímulo proporcionado pelas variações do nível do mar. Posteriormente, inúmeros autores visaram a avaliação de parâmetros hidrodinâmicos a partir da análise do efeito produzido por causas extra-aquífero (JACOB, 1940; FERRIS, 1951 - apud HUNTSMAN e MCCREADY, 1995); STALLMAN e PAPADOPULOS, 1966; GILMORE et al., 1992; CRUZ, 1997; MERRITT, 2004; FERNANDES et al., 2016; SÁNCHEZ-ÚBEDA et al., 2016; COSTA e BACELLAR, 2010; BARLOW e LEAKE, 2012).

No nordeste do Brasil, hidrogeólogos da antiga SUDENE, de empresas de consultoria e do Laboratório de Hidrogeologia da UFPE, sempre enfrentaram desafios para o dimensionamento de baterias de poços ou a definição da capacidade de produção de baterias de poços já existentes, em aquíferos regionais. A título de exemplo, citam-se os seguintes estudos:

- Aquífero Açu em Mossoró na Bacia Potiguar: Bezerra 
(1977, 1980); Caern/Hidrosolos (1970); Caern/Acquaplan (1972); Feitosa (1986, 1996, 1997); Filho (1968); Planat/Caern (1979).

- Aquífero Barreiras na região metropolitana de Natal: Caern/Planat (1979, 1980, 1981, 1983).

- Aquífero Beberibe na Região Metropolitana do Recife: França (1968); França e Capucci (1978); Oliveira (2003); Feitosa e Filho (2017).

- O Aquífero Cabeças no Vale do Gurguéia/PI: Feitosa et al. (1990); Feitosa e Demétrio (2009).

A avaliação das interferências em baterias de poços, nos casos acima citados, sempre constituiu um desafio que findou por induzir a concepção de modelos analíticos de fluxo (FEITOSA et al., 2008) para a solução satisfatória do que se convencionou chamar de cálculo de baterias de poços. É muito natural, assim, que as noções de raio de influência e difusividade tenham ocupado as atenções dos hidrogeólogos ligados a questões relativas a essas baterias. Dessa forma, não são utilizados nesse trabalho estímulos externos ao aquífero (oscilações das marés oceânicas, variações do nível de rios, curvas de recessão e variações da pressão atmosférica) para a avaliação das difusividades. Utilizam-se estímulos produzidos no próprio corpo do aquífero (bombeamentos de poços produtores) e a resposta a esses estímulos em poços de observação, após um tempo to designado de tempo de reação ("time lag" ou "delay time"). Essa abordagem foi utilizada por Beauheim (2007) e Ludvigson e Hjerne (2014) para meios heterogêneos. Aqui ela será adotada para um meio homogêneo de porosidade intergranular.

\subsection{Obtenção dos Dados}

Para a explicitação de D numa expressão analítica, em aquífero confinado, foram feitas utilizações convenientes da equação de Theis. O procedimento constou da geração de um grande número de pares $\left(D, t_{0}\right)$ os quais foram objeto de análises de regressão. Para a obtenção desses pares foi desenvolvido um programa de computador que cumpriu as seguintes tarefas:

- a) 0 programa toma a primeira vazão $\left(50 \mathrm{~m}^{3} / \mathrm{h}\right)$ e a primeira distância $\mathrm{r}(50 \mathrm{~m})$, conforme mostrado nas figuras 1 e 2.
- b) Em seguida, é gerado aleatoriamente um par teórico $(T, S)$, dentro de uma ampla gama de valores considerada realista, com o auxílio de um gerador de números aleatórios incorporado ao programa. Tendo esse par teórico (T, S), têm-se uma difusividade teórica e aleatória $D$.

- c) De posse desses valores, o programa calcula o rebaixamento num piezômetro fictício situado a $50 \mathrm{~m}$ do poço produtor (ver figuras 1 e 2). 0 cálculo é feito com utilização da equação de Theis (equação 2). Adota-se, inicialmente, um tempo de bombeamento igual a $10^{12}$ segundos para assegurar um rebaixamento inicial indubitavelmente maior que qualquer rebaixamento real. Como esse rebaixamento é maior que 0,0001 m (critério de convergência adotado), o tempo de bombeamento é diminuído de 0,1\% e um novo rebaixamento é calculado e comparado com o critério de convergência (Figura 2). Esse processo iterativo continua até que o rebaixamento calculado na distância de $50 \mathrm{~m}$ considerada e para a vazão de $50 \mathrm{~m}^{3} / \mathrm{h}$ (figuras 1 e 2) seja inferior a 0,0001 m, ou seja, sensivelmente próximo de zero. 0 tempo necessário para que isso ocorra é considerado como o to. Verifica-se, assim, que para a primeira vazão (50 $\mathrm{m}^{3} / \mathrm{h}$ ) e a primeira distância (50 m), têm-se ao final do processo iterativo, um par ( $D$, to). Como o programa executa 200 iterações para essa vazão e essa distância, têm-se, ao final das iterações, 200 pares (D, to). Esses pares são diferentes, pois que, a cada iteração, nova difusividade aleatória é gerada.

- d) O programa toma em seguida a segunda distância (100 m - figuras 1 e 2) e repete as operações acima descritas. Após a utilização da última distância (5.000 m - Figura 1), têm-se 3.000 pares (D, to) para a vazão de $50 \mathrm{~m}^{3} / \mathrm{h}$ (200 pares $\times 15$ distâncias), conforme indicado na Figura 1.

- e) 0 programa toma em seguida a segunda vazão (100,0 m³/h - Figura 1) e repete todas as operações descritas nos tópicos anteriores. Ao final do processo, isto é, após a utilização da sexta vazão (500 $m^{3} / h$ ), têm-se 18.000 pares ( $\left.D, t_{0}\right)$.

Figura 1 - Esquema de cálculo adotado

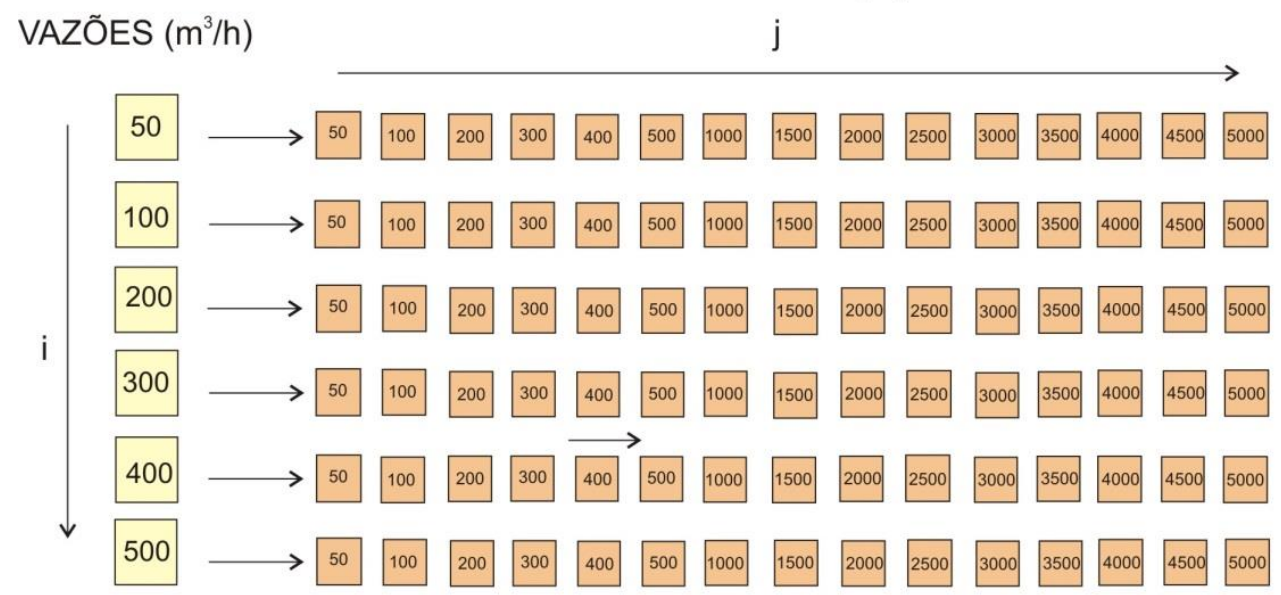

PARES $\left(\mathrm{t}_{0} \times \mathrm{T} / \mathrm{S}\right)$ GERADOS

200 pares $\left(\mathrm{t}_{0} \times \mathrm{T} / \mathrm{S}\right)$ por distância $=3000$ pares

200 pares $\left(\mathrm{t}_{0} \times \mathrm{T} / \mathrm{S}\right)$ por distância $=3000$ pares 200 pares $\left(\mathrm{t}_{0} \times \mathrm{T} / \mathrm{S}\right)$ por distância $=3000$ pares

200 pares $\left(\mathrm{t}_{0} \times \mathrm{T} / \mathrm{S}\right)$ por distância $=3000$ pares

200 pares $\left(\mathrm{t}_{0} \times \mathrm{T} / \mathrm{S}\right)$ por distância $=3000$ pares

200 pares $\left(\mathrm{t}_{0} \times \mathrm{T} / \mathrm{S}\right)$ por distância $=3000$ pares 


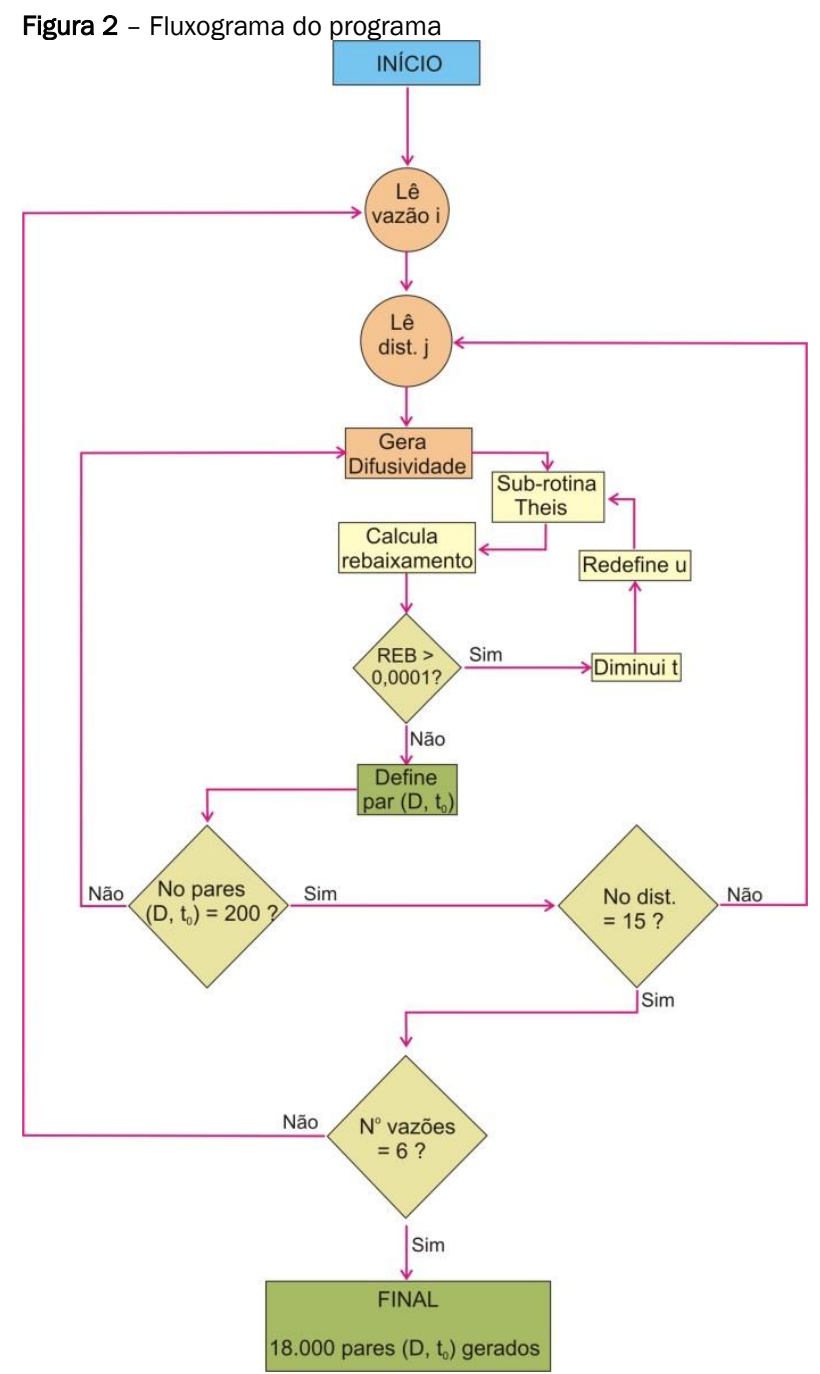

\subsection{Análise Estatística dos Dados}

A partir dos pares ( $\left.D, t_{0}\right)$, foi possível chegar a uma expressão analítica para a difusividade em função da distância $r$ do poço produtor a um poço de observação, e do tempo de reação to observado neste último. Para tal, foram realizados dois (02) conjuntos de correlações, descritos a seguir.

\subsubsection{Primeiro conjunto de correlações ( $\mathrm{D} \times \mathrm{to}$ )}

Para cada vazão, têm-se 15 conjuntos de 200 pares (D, to) escalonados segundo as distâncias ao poço bombeado. Esses conjuntos admitem ajustes de leis potenciais com coeficientes de determinação $\mathrm{R}^{2}$ nunca inferiores a 0,99. Na Figura 3, esses ajustes são mostrados para a vazão de $100 \mathrm{~m}^{3} / \mathrm{h}$.
A expressão matemática da lei, em diagrama log-log, para a vazão acima citada, é uma reta de equação:

$\ln D=B \ln t_{0}+C(r)$

em que:

D = difusividade hidráulica em $\mathrm{m}^{2} / \mathrm{s}$

to $\quad=$ tempo de reação, em minutos, do poço de observação situado a uma distância $r$, em metros, do poço bombeado.

B $=$ Inclinação da reta ajustada.

$\mathrm{C}(\mathrm{r})=$ Coeficiente linear da reta ou interseção com o eixo Y, dependente de r. 
Figura 3 - Leis Potenciais Ajustadas aos 15 Conjuntos de 200 Pares (D, to), para a vazão $Q=100 \mathrm{~m}^{3} / \mathrm{h}$ 1000000

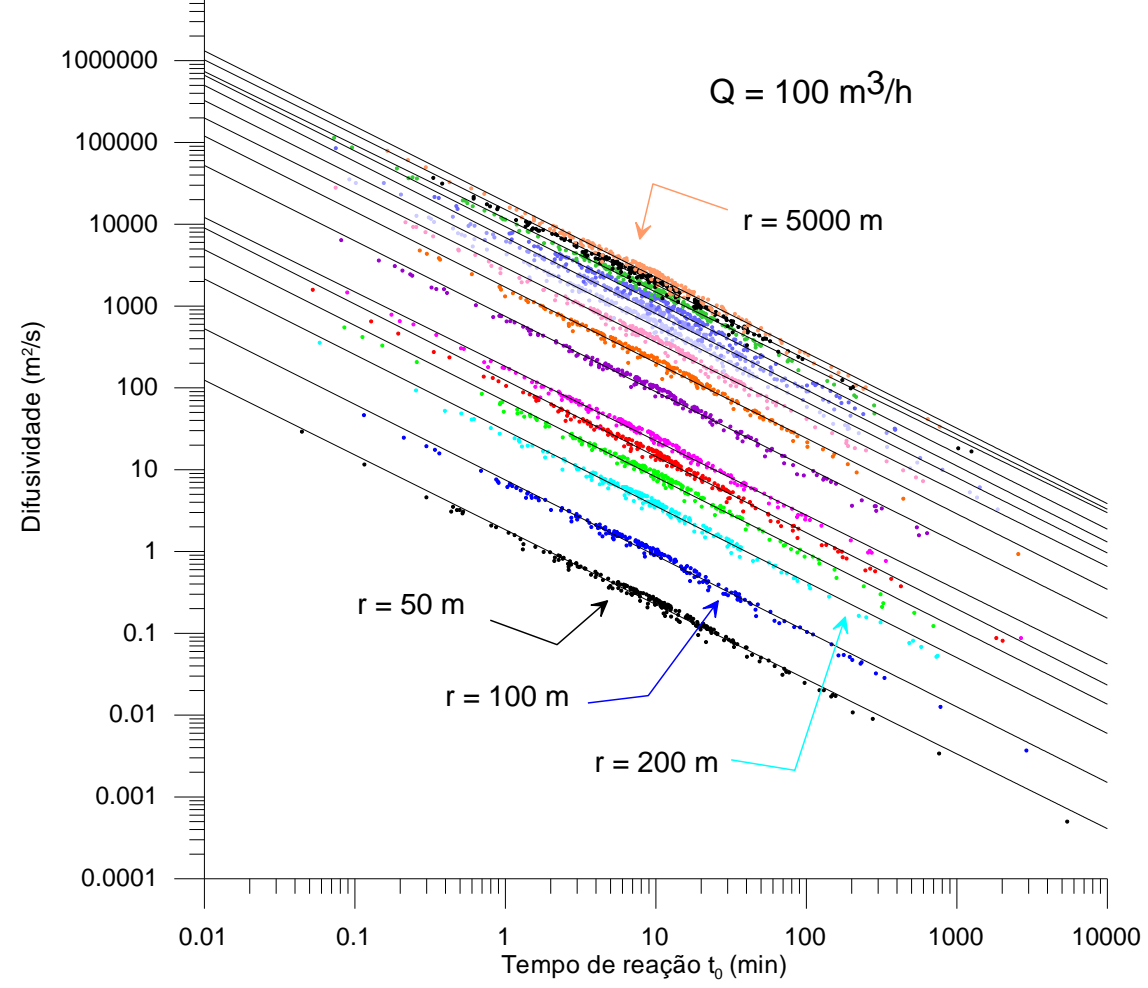


Tabela 1 - Intersecções e Inclinações das Retas (Leis Potenciais) Ajustadas aos 15 Conjuntos de 200 Pares (D, to).

\begin{tabular}{|c|c|c|c|c|c|c|c|c|c|c|c|c|c|c|c|c|}
\hline \multirow{2}{*}{$\begin{array}{l}\text { Vazões } \\
\left(\mathrm{m}^{3} / \mathrm{h}\right)\end{array}$} & \multirow{2}{*}{ Coeficientes da reta ajustada } & \multicolumn{15}{|c|}{ Distância do Poço de observação ao Poço Bombeado (m) } \\
\hline & & 50 & 100 & 200 & 300 & 400 & 500 & 1000 & 1500 & 2000 & 2500 & 3000 & 3500 & 4000 & 4500 & 5000 \\
\hline \multirow{2}{*}{50} & Intersecção (In D) & 0,716 & 2,134 & 3,468 & 4,338 & 4,858 & 5,407 & 6,707 & 7,573 & 8,150 & 8,608 & 8,942 & 9,275 & 9,547 & 9,732 & 9,949 \\
\hline & Inclinação (In D / In to) & $-0,903$ & $-0,914$ & $-0,896$ & $-0,913$ & $-0,889$ & $-0,931$ & $-0,906$ & $-0,923$ & $-0,923$ & $-0,926$ & $-0,923$ & $-0,924$ & $-0,928$ & $-0,916$ & $-0,921$ \\
\hline \multirow{2}{*}{100} & Intersecção (In D) & 0,614 & 2,011 & 3,408 & 4,229 & 4,817 & 5,213 & 6,618 & 7,441 & 7,993 & 8,450 & 8,836 & 9,146 & 9,371 & 9,622 & 9,844 \\
\hline & Inclinação (In D / In to) & $-0,914$ & $-0,924$ & $-0,926$ & $-0,926$ & $-0,931$ & $-0,910$ & $-0,922$ & $-0,924$ & $-0,914$ & $-0,922$ & $-0,930$ & $-0,925$ & $-0,899$ & $-0,915$ & $-0,923$ \\
\hline \multirow{2}{*}{200} & Intersecção (In D) & 0,535 & 1,921 & 3,291 & 4,115 & 4,669 & 5,103 & 6,527 & 7,320 & 7,907 & 8,323 & 8,725 & 9,013 & 9,288 & 9,519 & 9,729 \\
\hline & Inclinação (In D / In to) & $-0,932$ & $-0,934$ & $-0,925$ & $-0,932$ & $-0,920$ & $-0,914$ & $-0,933$ & $-0,919$ & $-0,928$ & $-0,915$ & $-0,930$ & $-0,922$ & $-0,927$ & $-0,922$ & $-0,920$ \\
\hline \multirow{2}{*}{300} & Intersecção (In D) & 0,468 & 1,850 & 3,255 & 4,037 & 4,628 & 5,079 & 6,465 & 7,273 & 7,851 & 8,312 & 8,660 & 8,991 & 9,257 & 9,478 & 9,677 \\
\hline & Inclinação (In D / In to) & $-0,929$ & $-0,928$ & $-0,932$ & $-0,926$ & $-0,934$ & $-0,935$ & $-0,929$ & $-0,940$ & $-0,933$ & $-0,937$ & $-0,939$ & $-0,938$ & $-0,947$ & $-0,935$ & $-0,938$ \\
\hline \multirow{2}{*}{400} & Intersecção (In D) & 0,459 & 1,819 & 3,231 & 4,032 & 4,616 & 5,036 & 6,435 & 7,241 & 7,810 & 8,250 & 8,642 & 8,928 & 9,212 & 9,451 & 9,656 \\
\hline & Inclinação (In D / In to) & $-0,943$ & $-0,939$ & $-0,949$ & $-0,940$ & $-0,945$ & $-0,932$ & $-0,939$ & $-0,937$ & $-0,937$ & $-0,932$ & $-0,943$ & $-0,933$ & $-0,939$ & $-0,941$ & $-0,940$ \\
\hline \multirow{2}{*}{500} & Intersecção (In D) & 0,426 & 1,809 & 3,191 & 4,007 & 4,584 & 5,006 & 6,402 & 7,220 & 7,800 & 8,243 & 8,629 & 8,884 & 9,178 & 9,419 & 9,621 \\
\hline & Inclinação (In D / In to) & $-0,945$ & $-0,942$ & $-0,943$ & $-0,948$ & $-0,945$ & $-0,934$ & $-0,945$ & $-0,945$ & $-0,939$ & $-0,941$ & $-0,950$ & $-0,929$ & $-0,939$ & $-0,942$ & $-0,939$ \\
\hline
\end{tabular}


Como foram consideradas seis vazões e obtidos 15 conjuntos de pares (D, to) por vazão, foram ajustadas no total $6 \times 15=90$ retas. As inclinações e as intersecções das retas ajustadas são mostradas na Tabela 1 , cabendo os seguintes comentários.

- A inclinação B das retas ajustadas se mantém dentro da estreita gama de $-0,90$ a $-0,95$. É possível assim considerar uma inclinação única e constante de -0,93.

- A intersecção $\mathrm{C}(\mathrm{r})$ das retas ajustadas, por outro lado, é uma função da distância $r$ do poço de observação ao poço bombeado.

Neste primeiro conjunto de correlações, portanto, foi obtida uma expressão (equação 8) que fornece o In $D$ em função do logaritmo neperiano do tempo de reação do poço de observação (to) e de uma função $C(r)$.

\subsubsection{Segundo conjunto de correlações $(C(r) \times r)$}

Como verificado acima, a intersecção $C(r)$ da equação 8 varia com a distância $r$ do poço de observação ao poço bombeado. Foram, então, realizadas seis correlações entre intersecção e distância, com 15 pontos cada uma (15 distâncias $r$ ). As seis correlações foram para as vazões de 50, 100, 200, 300, 400 e $500 \mathrm{~m}^{3} / \mathrm{h}$ (Figura 4) e tiveram o objetivo de explicitar $\mathrm{C}(\mathrm{r})$.

Os melhores ajustes conseguidos foram funções logarítmicas com coeficientes de determinação nunca inferiores a 0,99 . As expressões matemáticas das leis logarítmicas, em gráfico monolog, são retas de equação geral:

$\mathrm{Y}=\mathrm{aX}+\mathrm{b}$

Figura 4 - Gráficos $C(r)$ x r para seis vazões

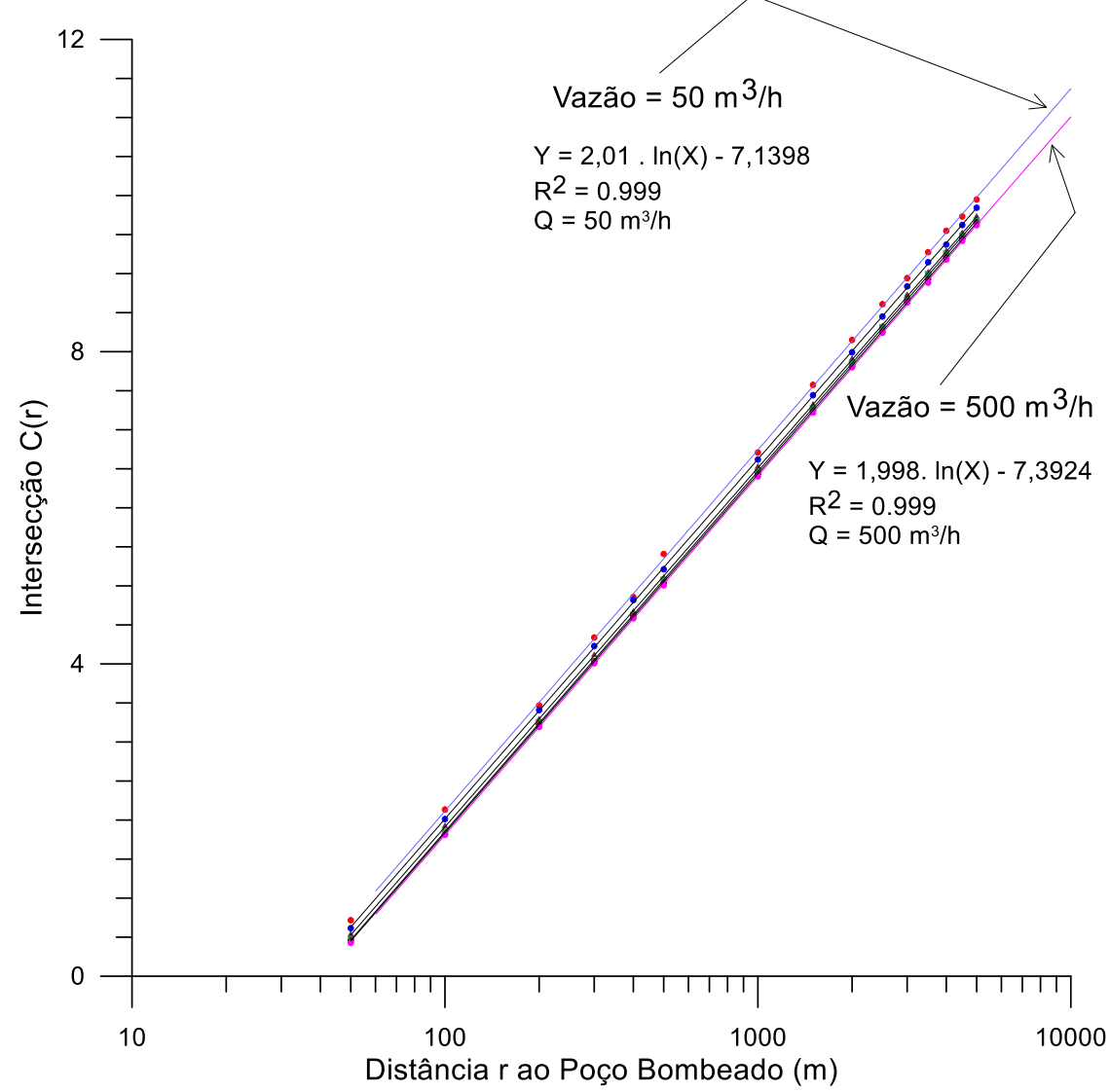


Nos seis gráficos obtidos verifica-se que os coeficientes angulares são constantes e sensivelmente iguais a 2. As intersecções, por sua vez (Tabela 2), podem ser também consideradas como uma constante, igual à média dos seis valores, 7,29 .

Tabela 2 - Vazões e intersecções $F(Q)$

\begin{tabular}{ll}
\hline $\mathrm{Q}\left(\mathrm{m}^{3} / \mathrm{h}\right)$ & $\mathrm{F}(\mathrm{Q})$ \\
\hline 50 & $-7,29$ \\
100 & $-7,20$ \\
200 & $-7,29$ \\
300 & $-7,37$ \\
400 & $-7,36$ \\
500 & $-7,39$ \\
\hline
\end{tabular}

Assim sendo, a equação geral (9) pode ser escrita como:

$C(r)=2,0 \ln r-7,29$

em que:

$\mathrm{C}(r)=$ interseção na equação (8).

$r=$ distância, em metros, do poço de observação ao poço bombeado.

\section{RESULTADOSEDISCUSSÃO}

As equações (8) a (10) permitem se chegar a uma expressão para a obtenção da difusividade hidráulica, conforme mostrado a seguir.

\subsection{Obtenção da Difusividade}

Na equação (8), a inclinação B é igual a -0,93. A intersecção $C(r)$, por sua vez, pode ser considerada como o logaritmo neperiano de um número A(r). Pode-se, então, escrever:

$A(r)=e^{C(r)}$

Levando-se em (11) o valor de $C(r)$ explicitado em (10), vem:

$A(r)=e^{(2 \ln r-7,29)}$

Considerando (11), a equação (8) pode ser escrita como:

$\ln D=B \ln t_{0}+\ln A(r) \quad$ ou

$\ln D=\ln \left(A(r) \cdot t_{0}{ }^{B}\right) \quad$ o que significa que

$D=A(r) \cdot t_{0}{ }^{B}$

Trazendo em (13) o valor de A(r) explicitado em (12) e o valor de $\mathrm{B}$, acima indicado, vem, finalmente:

$\mathrm{D}=\frac{\mathrm{e}^{(2 \ln -7,3)}}{\mathrm{t}_{0} 0,93}$
$\mathrm{D}=$ difusividade hidráulica em $\mathrm{m}^{2} / \mathrm{s}$

to $\quad=\quad$ tempo de reação, em minutos, do poço de observação situado a uma distância $r$, em metros, do poço produtor.

A equação (14) permite obter a difusividade hidráulica de um aquífero confinado conhecendo-se o tempo de reação, em minutos, de um poço de observação situado a $r$ metros do poço bombeado.

A difusividade é uma propriedade do meio poroso, dependente da transmissividade ( $\mathrm{T}$ ) e do coeficiente de armazenamento (S). Na equação (14) ela é expressa como uma relação não linear, entre a distância $r$ de um poço bombeado a um poço de observação e o tempo to necessário para que ocorra reação neste último, devida ao bombeamento ou à sua interrupção. Assim, por exemplo, para uma difusividade constante, um aumento da distância r corresponde a um aumento do tempo de reação to de acordo com a razão indicada na equação (14).

\subsubsection{Validação do Procedimento}

Na década de 1980 foram realizados 20 testes do Aquífero Cabeças/PI, confinado (FEITOSA et al., 1990), em três baterias de poços (bateria do Incra, bateria do Projeto Piloto e bateria da Unifor), incluindo um teste em um poço isolado (poço Tranqueira). A duração desses testes foi de 72 horas de bombeamento com vazão rigorosamente constante (320 m3/h a $600 \mathrm{~m}^{3} / \mathrm{h}$, dependendo do poço) aferida por escoador de orifício circular. Foi utilizada uma bomba turbina ESCO de eixo prolongado, pertencente à CPRM, acionada por motor MWM diesel de 12 cilindros. Os poços têm profundidades variando entre 350 e $466 \mathrm{~m}$, totalmente penetrantes no Aquífero Cabeças cujas espessuras variam entre 158 e $302 \mathrm{~m}$.

Desses 20 testes, 15 foram considerados no presente artigo. Como foram utilizados muitos poços de observação nesses testes, têm-se um total de 40 pares (T, S) e, consequentemente, 40 difusividades confiáveis para utilizar como referência.

Os dados referentes a estes testes são mostrados na Tabela 3 , onde as colunas têm os seguintes significados:

- Coluna C - Distâncias do poço bombeado ao poço observado.

- Colunas D e E - Parâmetros hidrodinâmicos obtidos dos testes de aquífero.

- Coluna F - Difusividades obtidas dos testes de aquífero $(D=T / S)$.

- Coluna G - Tempo de reação to do poço observa-

em que: 
do, obtido por extrapolação da curva de rebaixamento em gráfico bi-log, com auxílio da curva de Theis, até a ordenada de valor $\mathrm{s}_{\mathrm{w}}=0,001 \mathrm{~m}$.

- Coluna H - Difusividade D obtida pela aplicação da equação 14 para o tempo de reação to (coluna $\mathrm{G}$ ) e a distância $r$ (coluna $\mathrm{C}$ ).

- Coluna I-Difusividade D obtida pela aplicação da equação 7 para o tempo de reação to (coluna G) e a distância $r$ (coluna $C$ ). Os valores dessa coluna são irreais, extremamente elevados, demonstrando a impropriedade da equação (7), proposta por Custodio e Llamas (1983), para avaliar difusividades em poços de observação muito afastados do poço produtor.
Em todos os parâmetros que serão analisados a seguir e comparados entre si através de regressões, prevalece sempre a condição de contorno que estabelece que $Y=0$ para $X=0$. Por esta razão foram adotadas equações do tipo $Y=a X$ para as leis ajustadas aos dados experimentais. Estes são os pares (D, to) obtidos no item 2.2. e que são, em realidade, dados teóricos gerados com o auxílio da equação de Theis.

Na Figura 5 mostra-se a correlação entre as difusividades obtidas pela razão $\mathrm{T} / \mathrm{S}$ dos testes (coluna $\mathrm{F}$ na Tabela 3 ) e as difusividades obtidas pela equação 14 (coluna $\mathrm{H}$ na Tabela 3).

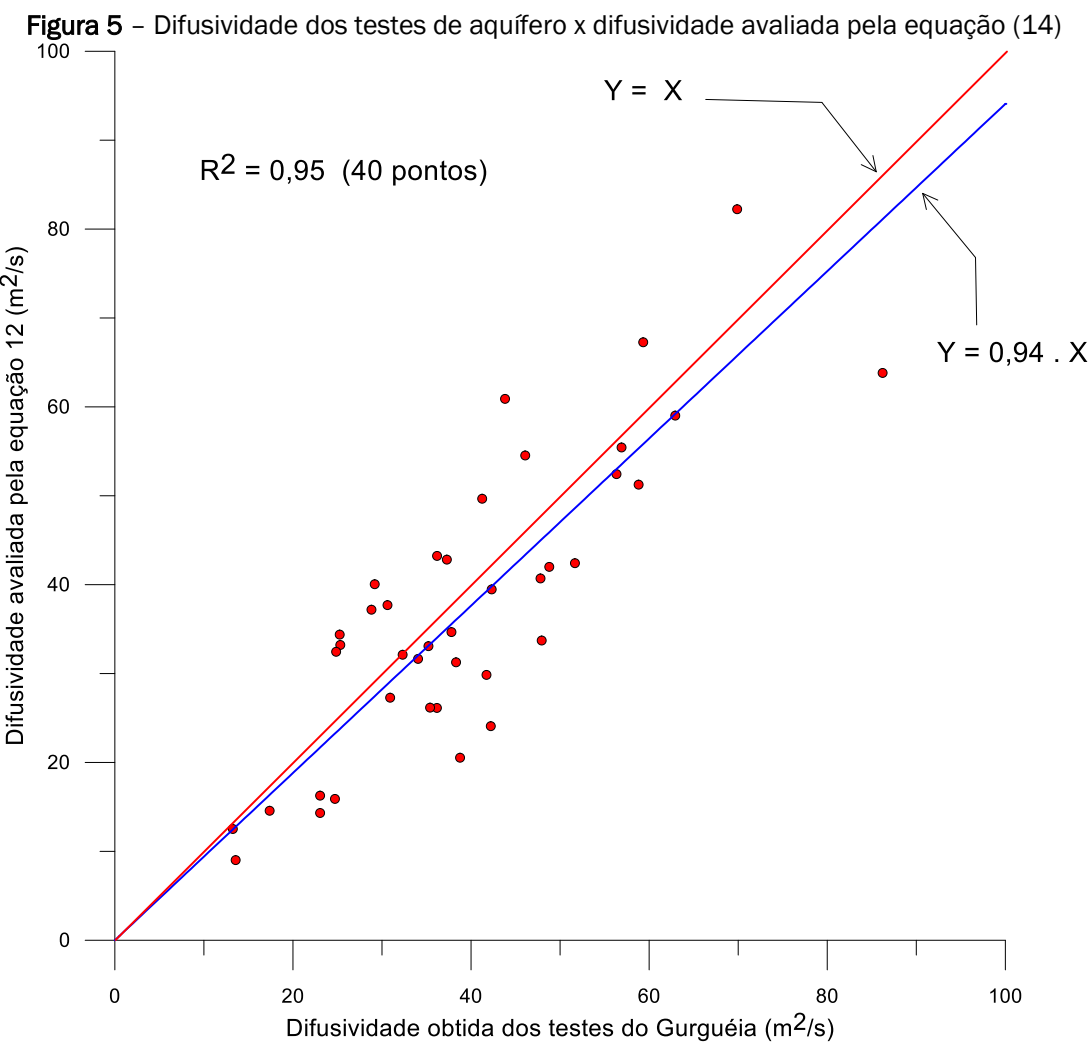


$\underline{\text { Tabela } 3 \text { - Difusividades avaliadas e seus confrontos com os testes das baterias do Gurguéia }}$

\begin{tabular}{|c|c|c|c|c|c|c|c|c|c|}
\hline Ordem & $\begin{array}{c}\text { A } \\
\text { Poço bombeado }\end{array}$ & $\begin{array}{c}\text { B } \\
\text { Poço } \\
\text { observado } \\
\end{array}$ & $\begin{array}{c}C \\
r(m)\end{array}$ & $\begin{array}{c}D \\
T\left(m^{2} / s\right)\end{array}$ & $\begin{array}{c}\text { E } \\
\text { S (Adim.) }\end{array}$ & $\begin{array}{c}F \\
D\left(m^{2} / \mathrm{s}\right) \\
(\mathrm{T} / \mathrm{S}) \\
\end{array}$ & $\begin{array}{c}G \\
t_{0}(\min )\end{array}$ & $\begin{array}{c}H \\
\text { D eq. } 14 \\
\left(\mathrm{~m}^{2} / \mathrm{s}\right) \\
\end{array}$ & $\begin{array}{c}\text { I } \\
\text { D1 eq.7 } \\
\left(\mathrm{m}^{2} / \mathrm{s}\right)\end{array}$ \\
\hline 1 & APp11 & APz8 & 1250 & 0,006730 & 0,000387 & 17,39 & 100,00 & 14,57 & 115,74 \\
\hline 2 & APp7 & APp6 & 910 & 0,012800 & 0,000267 & 47,94 & 20,50 & 33,71 & 299,22 \\
\hline 3 & APp6 & $\mathrm{APz} 7$ & 890 & 0,011300 & 0,000267 & 42,32 & 16,50 & 39,46 & 355,60 \\
\hline 4 & APp6 & APp5 & 1285 & 0,012000 & 0,000246 & 48,78 & 34,00 & 41,99 & 359,74 \\
\hline 5 & APp7 & APz4 & 2220 & 0,009750 & 0,000736 & 13,25 & 405,00 & 12,51 & 90,14 \\
\hline 6 & APp 7 & APz3 & 1250 & 0,027900 & 0,000728 & 38,32 & 44,00 & 31,27 & 263,05 \\
\hline 7 & APp 7 & APp2 & 1140 & 0,012400 & 0,000240 & 51,67 & 26,00 & 42,42 & 370,26 \\
\hline 8 & APp7 & APz7 & 20 & 0,010300 & 0,000244 & 42,21 & 0,01 & 24,09 & 370,37 \\
\hline 9 & APp6 & APz4 & 1320 & 0,038700 & 0,001070 & 36,17 & 60,00 & 26,13 & 215,11 \\
\hline 10 & APp6 & APz3 & 1940 & 0,012700 & 0,000393 & 32,32 & 110,00 & 32,12 & 253,44 \\
\hline 11 & APp6 & APp2 & 1210 & 0,015900 & 0,000268 & 59,33 & 18,00 & 67,27 & 602,51 \\
\hline 12 & APp6 & APz1 & 1180 & 0,013600 & 0,000239 & 56,90 & 21,00 & 55,43 & 491,15 \\
\hline 13 & APp6 & APp1 & 1185 & 0,016900 & 0,000300 & 56,33 & 22,50 & 52,43 & 462,30 \\
\hline 14 & APp5 & APz7 & 2165 & 0,006770 & 0,000235 & 28,81 & 119,00 & 37,18 & 291,77 \\
\hline 15 & APp 7 & APp5 & 2200 & 0,006640 & 0,000263 & 25,25 & 134,00 & 34,38 & 267,55 \\
\hline 16 & APp5 & APz1 & 1800 & 0,010600 & 0,000230 & 46,09 & 53,00 & 54,53 & 452,83 \\
\hline 17 & APp7 & APz1 & 1660 & 0,013000 & 0,000272 & 47,79 & 61,00 & 40,69 & 334,62 \\
\hline 18 & APp5 & APz4 & 470 & 0,045110 & 0,001274 & 35,41 & 6,50 & 26,17 & 251,74 \\
\hline 19 & APp4 & APz1 & 1150 & 0,011600 & 0,000166 & 69,88 & 13,00 & 82,24 & 753,56 \\
\hline 20 & APp4 & APz4 & 470 & 0,041200 & 0,001210 & 34,05 & 5,30 & 31,64 & 308,74 \\
\hline 21 & APp3 & APz3 & 20 & 0,010700 & 0,000433 & 24,71 & 0,01 & 15,91 & 237,04 \\
\hline 22 & APp2 & APz3 & 1090 & 0,019500 & 0,000445 & 43,82 & 16,00 & 60,91 & 550,05 \\
\hline 23 & APp1 & APz3 & 1930 & 0,051900 & 0,000602 & 86,21 & 52,00 & 63,81 & 530,61 \\
\hline 24 & APp1 & APz1 & 20 & 0,019830 & 0,000512 & 38,76 & 0,01 & 20,53 & 311,89 \\
\hline 25 & APp2 & APz1 & 840 & 0,015000 & 0,000255 & 58,82 & 11,00 & 51,25 & 475,15 \\
\hline 26 & Tranqueira & $\mathrm{T} 1$ & 220 & 0,009440 & 0,000150 & 62,93 & 0,53 & 59,01 & 676,45 \\
\hline 27 & Tranqueira & $\mathrm{T} 2$ & 990 & 0,007300 & 0,000177 & 41,24 & 16,20 & 49,67 & 448,15 \\
\hline 28 & BPp10 & BPz4 & 2170 & 0,011800 & 0,000466 & 25,32 & 135,00 & 33,22 & 258,38 \\
\hline 29 & P15 & BPz5 & 2305 & 0,012600 & 0,000338 & 37,28 & 117,00 & 42,81 & 336,37 \\
\hline 30 & BPp10 & BPz3 & 2720 & 0,009120 & 0,000367 & 24,85 & 225,00 & 32,45 & 243,57 \\
\hline 31 & BPp10 & BPz10 & 20 & 0,013546 & 0,000588 & 23,05 & 0,01 & 16,27 & 242,87 \\
\hline 32 & BPp2 & $\mathrm{BPz3}$ & 1135 & 0,011000 & 0,000291 & 37,80 & 32,00 & 34,66 & 298,20 \\
\hline 33 & BPp2 & BPz1 & 1255 & 0,010300 & 0,000353 & 29,18 & 34,00 & 40,06 & 343,14 \\
\hline 34 & BPp2 & BPz2 & 20 & 0,013600 & 0,000440 & 30,91 & 0,01 & 27,28 & 423,28 \\
\hline 35 & BPp1 & BPz1 & 145,02 & 0,017385 & 0,000417 & 41,73 & 0,45 & 29,86 & 346,19 \\
\hline 36 & CPpVII & CPz14 & 2710 & 0,015400 & 0,000503 & 30,62 & 190,00 & 37,70 & 286,32 \\
\hline 37 & CPpVII & $\mathrm{CPzVII}$ & 20 & 0,013300 & 0,000980 & 13,57 & 0,02 & 9,02 & 128,82 \\
\hline 38 & CPp7 & CPp6 & 2600 & 0,015200 & 0,000420 & 36,19 & 150,00 & 43,23 & 333,83 \\
\hline 39 & CPp7 & $\mathrm{CPz} 7$ & 20 & 0,013546 & 0,000588 & 23,05 & 0,01 & 14,32 & 211,64 \\
\hline 40 & CPp6 & $\mathrm{CPz} 7$ & 2600 & 0,015000 & 0,000426 & 35,21 & 200,00 & 33,09 & 250,37 \\
\hline
\end{tabular}

Existe uma boa correlação entre os dois conjuntos de difusividade $\left(R^{2}=0,95\right)$. Observa-se uma discreta subestimação $(\mathrm{Y}=0,94$. $\mathrm{X})$ dos valores da difusividade avaliados pela equação (14) em relação àqueles obtidos a partir dos tes tes de aquífero. Isso pode ser explicado pela discreta superestimação dos tempos to resultante da forma como foram obtidos. Esses tempos, com efeito, foram tomados na curva de Theis para um rebaixamento de 0,001 m, en- 
quanto no programa que gerou os pares teóricos (D, to), discutidos no item 2.2, foram considerados rebaixamentos menores que 0,0001 $\mathrm{m}$ como limite das aproximações sucessivas.

\subsection{Explicitação do par (T, S) a partir da difusividade}

Uma vez obtidas as difusividades $D$ para cada par poço bombeado/poço observado, é possível explicitar seus respectivos parâmetros $(\mathrm{T}, \mathrm{S})$ a partir do procedimento analítico mostrado a seguir:

A equação (2) aplicada a um poço de observação qualquer pode ser escrita como:

$s^{\prime}=\frac{Q}{4 \pi T} W(U)$

em que:

$$
\begin{aligned}
& \mathrm{s}^{\prime} \quad=\quad \text { rebaixamento num dado tempo } \\
& \mathrm{t}^{\prime}(\mathrm{m}) \\
& \mathrm{Q}=\text { vazão constante de bombea- } \\
& \text { mento }\left(\mathrm{m}^{3} / \mathrm{h}\right) \\
& \mathrm{T}=\text { transmissividade hidráulica } \\
& \left(\mathrm{m}^{2} / \mathrm{s}\right) \\
& \mathrm{W}(\mathrm{u})=\text { função de Theis explicitada na } \\
& \text { equação (3) } \\
& \mathrm{u}=\mathrm{r}^{2} \mathrm{~S} / 4 \mathrm{Tt} \text { em que: }
\end{aligned}
$$

A expressão para u pode ser convenientemente escrita como:

$u=\frac{r^{2}}{4 t^{\prime}} \cdot \frac{s}{T}$

em que:

$$
\begin{aligned}
r= & \text { distância do poço bombeado ao poço de } \\
& \text { observação }(\mathrm{m})
\end{aligned}
$$

Na equação (16) todas as variáveis são conhecidas, sendo $(\mathrm{S} / \mathrm{T})=1 / \mathrm{D}$ o inverso da difusividade que foi avaliada pela equação (14). De posse do valor de u para cada par poço bombeado/poço observado, calcula-se o respectivo $\mathrm{W}(\mathrm{u})$ com auxílio de uma rotina computacional conveniente.

Manipulando-se convenientemente a equação (15), chega-se à seguinte expressão:

$\mathrm{T}=\frac{\mathrm{QW}(\mathrm{u})}{4 \Pi \mathrm{s}^{\prime}}$

A equação (17) fornece a transmissividade para cada par poço bombeado/poço observado, com auxílio de uma rotina computacional conveniente. Essa mesma rotina fornece os respectivos coeficientes de armazenamento utilizando a equação (18).

$S=T / D$

Os pares (t', s') foram tomados nas curvas de rebaixamento dos testes de aquífero do Gurguéia (FEITOSA et al., 1990) de modo que os valores s' fossem no mínimo centimétricos para minimizar as imprecisões. Esse procedimento acarretou tempos de bombeamento t' variando entre 5 minutos e 14 horas, dependendo da distância r.

Observe-se que, teoricamente, qualquer ponto (t', s') da curva de rebaixamento fornece de forma unívoca o par ( $\mathrm{T}$, S), desde que a difusividade seja conhecida.

\subsubsection{Validação do Procedimento}

Tal como feito na obtenção das difusividades, a validação da abordagem para a obtenção dos pares (T, S) aqui discutidos foi feita considerando como referência os pares ( $T$, S) obtidos a partir dos testes de aquífero realizados no Aquífero Cabeças, Vale do Gurguéia/PI (FEITOSA et al., 1990) (Tabela 4)

Na Figura 6 mostra-se a correlação entre as transmissividades obtidas dos testes de aquífero do Aquífero Cabeças e as transmissividades fornecidas pela equação (17). 0 coeficiente de determinação $R^{2}=0,94$ sugere que estas últimas são aceitáveis como representativas do aquífero em questão.

Na Figura 7 mostra-se a correlação entre os coeficientes de armazenamento obtidos dos testes de aquífero do Aquífero Cabeças e os coeficientes de armazenamento fornecidos pela equação (18). 0 coeficiente de determinação $\mathrm{R}^{2}=0,97$ sugere que estes últimos são também aceitáveis como representativos do aquífero em questão. 
Tabela 4 - Dados utilizados, pares $(T, S)$ avaliados e seus confrontos com os pares $(T, S)$ avaliados nos testes das baterias do Gurguéia

(continua)

\begin{tabular}{|c|c|c|c|c|c|c|c|c|c|}
\hline Ordem & $\begin{array}{c}\text { Poço } \\
\text { produtor }\end{array}$ & $\begin{array}{c}\text { Poço de } \\
\text { observação }\end{array}$ & $\begin{array}{c}\text { D obtida pela equação } \\
(14)\left(\mathrm{m}^{2} / \mathrm{s}\right)\end{array}$ & $\mathbf{s}^{\prime}(\mathrm{m})$ & $\mathrm{t}^{\prime}(\min )$ & $\begin{array}{c}\text { Tavaliada a } \\
\text { partir da } \mathrm{D}\left(\mathrm{m}^{2} / \mathrm{s}\right)\end{array}$ & $\begin{array}{c}\text { Tobtido a partir dos testes } \\
\text { de aquífero }\left(\mathrm{m}^{2} / \mathrm{s}\right)\end{array}$ & $\begin{array}{c}\text { S avaliado a partir da } \\
\text { D (adim) }\end{array}$ & $\begin{array}{c}\text { S obtido a partir dos testes de } \\
\text { aquífero (adim) }\end{array}$ \\
\hline 1 & APp11 & APz8 & 14,57 & 0,201 & 360 & 0,00522398 & 0,00673000 & 0,00035854 & 0,00038700 \\
\hline 2 & APp7 & APp6 & 33,71 & 0,328 & 150 & 0,00861490 & 0,01280000 & 0,00025556 & 0,00026700 \\
\hline 3 & APp6 & APz7 & 39,46 & 0,600 & 240 & 0,01081850 & 0,01130000 & 0,00027416 & 0,00026700 \\
\hline 4 & APp6 & APp5 & 41,99 & 0,399 & 300 & 0,01035393 & 0,01200000 & 0,00024658 & 0,00024600 \\
\hline 5 & APp7 & APz4 & 12,51 & 0,043 & 790 & 0,00746792 & 0,00975000 & 0,00059696 & 0,00073600 \\
\hline 6 & APp7 & APz3 & 31,27 & 0,098 & 245 & 0,02119338 & 0,02790000 & 0,00067776 & 0,00072800 \\
\hline 7 & APp7 & APp2 & 42,42 & 0,880 & 685 & 0,01064043 & 0,01240000 & 0,00025084 & 0,00024000 \\
\hline 8 & APp7 & APz7 & 24,09 & 3,618 & 10 & 0,00891636 & 0,01030000 & 0,00037013 & 0,00024400 \\
\hline 9 & APp6 & APz4 & 26,13 & 0,137 & 485 & 0,02853370 & 0,03870000 & 0,00109199 & 0,00107000 \\
\hline 10 & APp6 & APz3 & 32,12 & 0,040 & 250 & 0,01062604 & 0,01270000 & 0,00033082 & 0,00039300 \\
\hline 11 & APp6 & APp2 & 67,27 & 0,149 & 125 & 0,01946589 & 0,01590000 & 0,00028937 & 0,00026800 \\
\hline 12 & APp6 & APz1 & 55,43 & 0,494 & 300 & 0,01312686 & 0,01360000 & 0,00023682 & 0,00023900 \\
\hline 13 & APp6 & APp1 & 52,43 & 0,334 & 265 & 0,01629005 & 0,01690000 & 0,00031070 & 0,00030000 \\
\hline 14 & APp5 & $\mathrm{APz} 7$ & 37,18 & 0,200 & 500 & 0,01228226 & 0,00677000 & 0,00033035 & 0,00023500 \\
\hline 15 & APp 7 & APp5 & 34,38 & 0,214 & 725 & 0,01043734 & 0,00664000 & 0,00030359 & 0,00026300 \\
\hline 16 & APp5 & APz1 & 54,53 & 0,318 & 370 & 0,01518773 & 0,01060000 & 0,00027852 & 0,00023000 \\
\hline 17 & APp7 & APz1 & 40,69 & 0,566 & 550 & 0,00702922 & 0,01300000 & 0,00017275 & 0,00027200 \\
\hline 18 & APp5 & APz4 & 26,17 & 0,402 & 180 & 0,03762327 & 0,04900000 & 0,00143765 & 0,00127400 \\
\hline 19 & APp4 & APz1 & 82,24 & 0,844 & 230 & 0,01357934 & 0,01160000 & 0,00016512 & 0,00016600 \\
\hline 20 & APp4 & APz4 & 31,64 & 0,387 & 150 & 0,03984573 & 0,04120000 & 0,00125935 & 0,00121000 \\
\hline 21 & APp3 & APz3 & 15,91 & 4,019 & 5 & 0,00902761 & 0,01070000 & 0,00056742 & 0,00043300 \\
\hline 22 & APp2 & APz3 & 60,91 & 0,433 & 250 & 0,02594045 & 0,01950000 & 0,00042588 & 0,00044500 \\
\hline 23 & APp1 & APz3 & 63,81 & 0,125 & 360 & 0,03416615 & 0,05190000 & 0,00053544 & 0,00060200 \\
\hline 24 & APp1 & APz1 & 20,53 & 3,069 & 15 & 0,01651546 & 0,01983002 & 0,00080446 & 0,00051161 \\
\hline 25 & APp2 & APz1 & 51,25 & 0,470 & 100 & 0,01352672 & 0,01500000 & 0,00026394 & 0,00025500 \\
\hline
\end{tabular}


Tabela 4 - Dados utilizados, pares ( $T, S$ ) avaliados e seus confrontos com os pares ( $T, S$ ) avaliados nos testes das baterias do Gurguéia

(conclusão)

\begin{tabular}{|c|c|c|c|c|c|c|c|c|c|}
\hline Ordem & $\begin{array}{l}\text { Poço } \\
\text { produtor }\end{array}$ & $\begin{array}{l}\text { Poço de } \\
\text { observação }\end{array}$ & $\begin{array}{l}\text { D obtida pela equação } \\
(14)\left(m^{2} / s\right)\end{array}$ & $\mathbf{s}^{\prime}(\mathrm{m})$ & $\mathrm{t}^{\prime}(\min )$ & $\begin{array}{c}\text { T avaliada a } \\
\text { partir da } D\left(\mathrm{~m}^{2} / \mathrm{s}\right)\end{array}$ & $\begin{array}{c}\text { Tobtido a partir dos testes } \\
\text { de aquífero }\left(\mathrm{m}^{2} / \mathrm{s}\right)\end{array}$ & $\begin{array}{c}\text { S avaliado a partir da } \\
\text { D (adim) }\end{array}$ & $\begin{array}{c}\text { S obtido a partir dos testes de } \\
\text { aquífero (adim) }\end{array}$ \\
\hline 26 & Tran & T1 & 59,01 & 2,614 & 70 & 0,00954080 & 0,00944000 & 0,00016168 & 0,00015000 \\
\hline 27 & Tran & T2 & 49,67 & 0,687 & 185 & 0,00923093 & 0,00730000 & 0,00018585 & 0,00017700 \\
\hline 28 & BPp10 & BPz4 & 33,22 & 0,160 & 675 & 0,01937133 & 0,01180000 & 0,00058312 & 0,00046600 \\
\hline 29 & P15 & BPz5 & 42,81 & 0,102 & 410 & 0,01672002 & 0,01260000 & 0,00039056 & 0,00033800 \\
\hline 30 & BPp10 & BPz3 & 32,45 & 0,143 & 840 & 0,01410760 & 0,00912000 & 0,00043475 & 0,00036700 \\
\hline 31 & BPp10 & BPz10 & 16,27 & 5,365 & 180 & 0,01465567 & 0,01581674 & 0,00090078 & 0,00057889 \\
\hline 32 & BPp2 & BPz3 & 34,66 & 0,232 & 180 & 0,01076105 & 0,01100000 & 0,00031048 & 0,00029100 \\
\hline 33 & BPp2 & BPz1 & 40,06 & 0,625 & 480 & 0,01163178 & 0,01030000 & 0,00029036 & 0,00035300 \\
\hline 34 & BPp2 & BPz2 & 27,28 & 3,083 & 10 & 0,01314499 & 0,01360000 & 0,00048185 & 0,00044000 \\
\hline 35 & BPp1 & BPz1 & 29,86 & 1,337 & 27 & 0,01452297 & 0,01738531 & 0,00048637 & 0,00041664 \\
\hline 36 & CPpVII & CPz14 & 37,70 & 0,091 & 852 & 0,01969669 & 0,01540000 & 0,00052246 & 0,00050300 \\
\hline 37 & CPpVII & $\mathrm{CPzVII}$ & 9,02 & 2,490 & 10 & 0,01039663 & 0,01330000 & 0,00115262 & 0,00098000 \\
\hline 38 & $\mathrm{CPp} 7$ & CPp6 & 43,23 & 0,171 & 792 & 0,01936908 & 0,01520000 & 0,00044805 & 0,00042000 \\
\hline 39 & CPp7 & $\mathrm{CPz} 7$ & 14,32 & 3,063 & 10 & 0,01409877 & 0,01354593 & 0,00098455 & 0,00058762 \\
\hline 40 & CPp6 & $\mathrm{CPz} 7$ & 33,09 & 0,150 & 855 & 0,01469595 & 0,01500000 & 0,00044412 & 0,00042600 \\
\hline
\end{tabular}


Figura 6 - Comparação entre transmissividades obtidas dos testes de aquífero e transmissividades avaliadas via difusividade

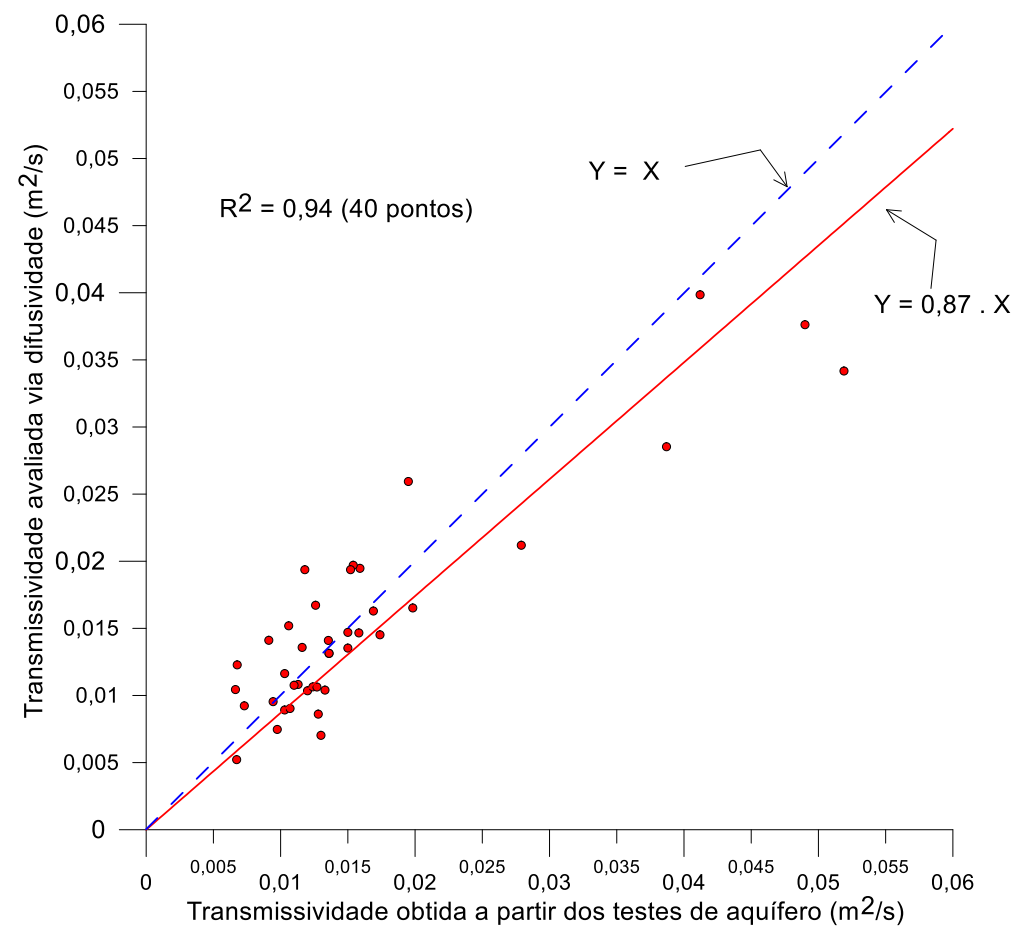

Figura 7 - Comparação entre coeficientes de armazenamento obtidos dos testes de aquífero e coeficientes de armazenamento avaliados via difusividade

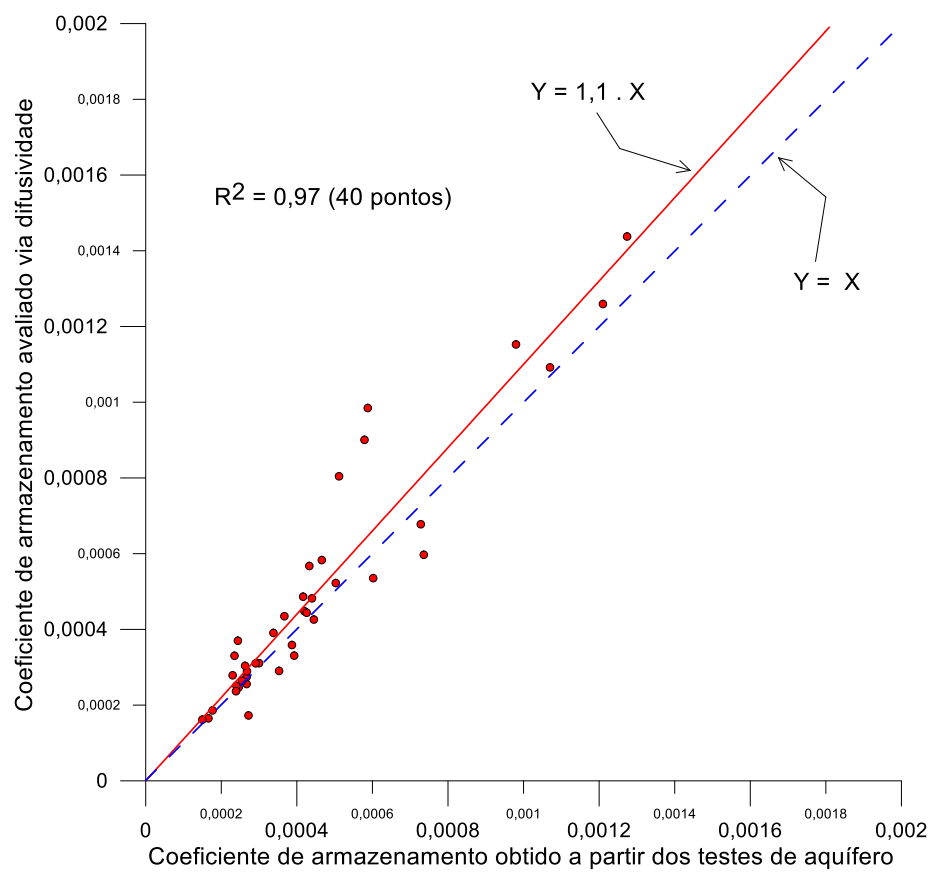

\section{CONCLUSÕESERECOMENDAÇÕES}

É proposto no trabalho um método para obtenção da difusividade hidráulica de aquíferos porosos confinados e, a partir desta, dos parâmetros transmissividade hidráulica (T) e coeficiente de armazenamento (S). As características essenciais do método proposto são as seguintes:
- A difusividade, em $\mathrm{m}^{2} / \mathrm{s}$, pode ser obtida pela equação $D=\frac{e^{(2 \ln r-7,3)}}{t_{0} 0,93}(14)$ em que to é o tempo de reação, em minutos, de um poço de observação situado a uma distância r, em metros, de um poço bombeando uma vazão qualquer. 
- A equação (14) é válida dentro de uma ampla gama de difusividades. As correlações realizadas, com efeito, utilizaram valores teóricos escalonados entre 0,012 m²/s e $8.914,0 \mathrm{~m}^{2} / \mathrm{s}$. Dentro dessa gama situam-se comodamente as difusividades realistas variando entre $13,0 \mathrm{~m}^{2} / \mathrm{s}$ e $86,0 \mathrm{~m}^{2} / \mathrm{s}$ (coluna F da Tabela 3) avaliadas nos poços do Vale do Gurguéia/PI.

- $\quad$ O tempo de reação to, observado num dado poço de observação, é válido para um poço produtor que iniciou ou que interrompeu o bombeamento.

- A partir da difusividade obtida com a equação (14) pode-se avaliar a transmissividade e o coeficiente de armazenamento de forma unívoca, utilizando-se as equações (17) e (18).

As conclusões acima estabelecidas autorizam as seguintes recomendações:

- Os valores de to, t' e s' utilizados na avaliação do $\operatorname{par}(T, S)$ foram obtidos dos testes de aquífero do Aquífero Cabeças, Gurguéia/PI. Em futuras aplicações práticas do método, entretanto, devem ser adotados procedimentos específicos que devem melhorar a precisão dos resultados. Esses procedimentos são os seguintes:

> Instalar no poço de observação um sensor transdutor de pressão que vai monitorar continuamente as variações do nível d'água. Na impossibilidade dessa instalação, pode-se utilizar um medidor de nível convencional.

> Anotar como tempo zero o momento da ligação ou desligamento da bomba do poço produtor.

$>0$ tempo to, em minutos, será o tempo que marca a reação do poço de observação. Essa reação deve se caracterizar por um aumento brusco do nível d'água ou por uma recuperação brusca desse nível.

> Manter a observação até a obtenção de um valor considerado confiável de rebaixamento ou recuperação. Esse valor será o s' acima citado e o tempo correspondente será o t'. Para poços de observação muito distantes do poço bombeado (2.500,0 m, por exemplo) o t' pode chegar a 14 horas como verificado no Gurguéia. Para distâncias mais razoáveis, de 200,0 ou 300,0 m, entretanto, esse tempo se reduz para algo em torno dos 5 minutos.

- $\quad$ A equação (14) aqui apresentada é válida para aquífero confinado. É sempre possível, entretanto, obter equações análogas para aquíferos semiconfinados ou livres adotando-se a mesma linha de raciocínio, mas, utilizando-se soluções analíticas da equação geral do fluxo subterrâneo (equação 1) adaptada às respectivas situações.

A abordagem aqui defendida não substitui os clássicos testes de aquífero. Num campo complexo de bombeamento, entretanto, onde poços entram e saem de operação frequentemente, como no caso do Aquífero Beberibe na região metropolitana norte do Recife, é praticamente impossivel a realização desses testes. Nessas situações, a aplicação consciente da abordagem aqui discutida pode fornecer valores da transmissividade $(T)$ e do coeficiente de armazenamento (S) suficientemente representativos do aquífero de modo a permitir projetos adequados de poços e de baterias de poços.

\section{AGRADECIMENTOS}

Agradeço ao hidrogeólogo Prof. José Geilson Alves Demétrio pela revisão do texto e, principalmente, pelas discussões e sugestões quando da concepção da abordagem. Agradeço também aos hidrogeólogos Helena Magalhães Porto Lira, João Manoel Filho e Fernando Antonio Carneiro Feitosa pela revisão do texto.

\section{REFERÊNCIAS}

ANA. Avaliação dos recursos hídricos subterrâneos e proposição de modelo de gestão compartilhada para os aquíferos da Chapada do Apodi, entre os estados do Rio Grande do Norte e Ceará. Relatório Final, Vol. III - Hidrogeologia, 151 p, 2010.

FEITOSA, E.C.; DEMÉTRIO, J.G.A. Os Aquíferos Cabeças e Serra Grande no Vale do Gurgéia - PI: síntese dos conhecimentos e perspectivas de explotação. Labhid/CTG/UFPE - CPRM, relatório inédito, Recife, 58 p, 2009.

FEITOSA, E.C.; FILHO, J.M. O Aquífero Beberibe na Área do Botafogo - Município de Itapissuma/PE: Viabilidade de uma bateria de poços. Atepe/Pöyry Tecnologia Ltda, relatório inédito, Recife, 20 p, 2017.

FEITOSA, E.C. (Coord.); COSTA, W.D; DEMÉTRIO, J.G.A.; FEITOSA, F.A.C. O Aquífero Cabeças no Vale do Gurguéia: Atualização dos Conhecimentos - Relatório Final (03 volumes). UFPE/ATEPE/DNOCS, Recife. Relatório Inédito, 204 p, 1990.

FEITOSA, E.C. A Explotação do Aqüífero Açu na Região de Mossoró - Caracterização da Situação Atual e Perspectivas de Atendimento da Demanda Futura. CPRM, Série Hidrogeologia: Pesquisa e Desenvolvimento, v.1, 44 p.il, Brasília, 1996.

FEITOSA, E.C. Captação de Mossoró: perspectivas de atendimento da demanda futura e previsão de níveis dinâmicos. CAERN/ATEPE, Natal, relatório inédito, 66 p, 1986.

FEITOSA, E.C. Parecer sobre as causas do aprofundamento dos níveis de bombeamento em Mossoró. SERHID/RN - Secretaria de Recursos Hídricos e Projetos Especiais do Rio Grande do Norte. Natal, Relatório Inédito, $20 \mathrm{p}, 1997$. 
BARLOW, P.M.; LEAKE, S.A. Streamflow depletion by wells - Understanding and Managing the Effects of Groundwater Pumping on Streamflow. U.S. Department of the Interior, Geological Survey, Groundwater Resources Program, Circular 1376, 2012.

BEAUHEIM, R.L. Diffusivity mapping of fracture interconnections. Conference: 2007 U.S. EPA/NGWA Fractured Rock Conference: State of the Science and Measuring Success in Remediation, Portland, Maine, p. 235-249, 2007.

BEZERRA, M.A. Captação de Mossoró: diagnóstico sobre a situação do manancial e do dispositivo de captação. CAERN/PLANAT, Natal, relatório inédito, 50 p, 1980.

BEZERRA, M.A. Captação de Mossoró: dimensionamento das condições de exploração dos poços tubulares e programa de trabalho complementar. CAERN/PLANAT, Natal, relatório inédito, 45 p, 1977.

CAERN/ACQUAPLAN. Projeto de Abastecimento d'Água de Mossoró/RN: estudo do manancial subterrâneo. Recife, relatório inédito, 47 p, 1972.

CAERN/HIDROSOLOS. Estudos Hidrogeológicos para Ampliação do Abastecimento d'Água da Cidade de Mossoró/RN. Recife, relatório inédito, 52 p, 1970.

CAERN/PLANAT. Captação de cidade satélite: poços produtores - relatório de conclusão. Natal/RN, relatório inédito, 45 p,1983.

CAERN/PLANAT. Captação de encanto: poços produtores relatório de conclusão. Natal/RN, relatório inédito, $37 \mathrm{p}$, 1980.

CAERN/PLANAT. Captação de ielmo marinho: poços produtores - relatório de conclusão. Natal/RN, relatório inédito, 29 p, 1981.

CAERN/PLANAT. Captação do conjunto Pirangi: poços produtores - relatório de conclusão. Natal/RN, relatório inédito, 40 p, 1979.

COOPER, H.H.; JACOB, C.E. A generalized graphical method for evaluating formation constants and summarizing well field history. Transactions of the American Geophysical Union, v. 27, p. 526-534, 1946.

COSTA, F.M.; BACELLAR, L.A.P. Caracterização hidrogeológica de aquíferos a partir do fluxo de base. RBRH - Águas Subterrâneas, v. 15, n. 3, p. 173-183, 2010.

CRUZ, J.V. Estudo hidrogeológico da Ilha do Pico (Açores Portugal). Tese (Doutorado em Geologia) - Universidade dos Açores, v. 2, Ponta Delgada, 433p, 1997.

CUSTODIO E.; LLAMAS, M.R. Hidrologia subterranea. 2. ed Edicionnes Omega S.A., Barcelona, v. 1, 1157 p, 1983.

FEITOSA, F.A.C; FILHO, J.M.; FEITOSA, E.C; DEMÉTRIO, J.G.A., (Coordenadores). Hidrogeologia: Conceitos e Aplicações. 3. ed. CPRM/LABHID-UFPE, 2008. 812 p,

FERNANDES, M.F.S.; CASTRO, J.W.A.; SILVA Jr, G.C.; MENDES, G.S. Estimativa de Parâmetros Hidrodinâmicos do
Aquífero Emborê em Farol de São Tomé - RJ com as oscilações do nível piezométrico e maré oceânica. Águas Subterrâneas, v. 30, n. 2, p. 209-223, 2016.

FERRIS, J.G. Cyclic fluctuations of water level as a basis for determining aquifer transmissibility: Internat. Geodesy Geophysics Union, Assoc. Sci. Hydrology Gen. Assembly, Brussels, v. 2, p. 148-155; duplicated 1952 as U. S. Geological survey Ground Water Note 1, 1951.

FETTER, C.W. Applied hydrogeology. 3. ed. New York: Macmillan Publishing Company, 1994.

FILHO, J.M. (Coord.). Bacia potiguar: estudo por analogia elétrica das condições de exploração das águas subterrâneas do arenito açú na região de Mossoró/RN. SUDENE, DD, Série Hidrogeologia n²3, Recife, 78 p,1968.

FRANÇA, H.P.M.; CAPUCCI, E.B. Diagnóstico preliminar das condições de exploração de água subterrânea do aquífero Beberibe - área Olinda-Paulista-Itamaracá. CPRM / COMPESA - Recife, relatório inédito, 75 p, 1978.

FRANÇA, H.P.M. Diagnóstico preliminar das condições de explotação de água subterrânea no aquífero Beberibe, área Olinda, Paulista e Itamaracá. CPRM. Recife, relatório inédito, 68 p, 1968.

GILMORE, T.J.; SPANE Jr, E.A.; NEWCOMER, D.R.; SHERWOOD, C.R. Application of Three Aquifer Test Methods for Estimating Hydraulic Properties Within the 100-N Area. Pacific Northwest Laboratory, Richland, Washington 99352, 49 P, 1992

HUNTSMAN, B.E.; MCCREADY, R.W. Passive Monitoring to Determine Aquifer Diffusivity. International Association of Hydrogeologists Congress. Edmonton, Alberta, Canada, 1995.

JACOB, C.E. Flow of groundwater in engineering hydraulics, edited by H. Rouse, New York. John Wiley and Sons, 1950.

JACOB, C.E. On the flow of water in an elastic artesian aquifer: American Geophysical Union Transactions. part 2, $p$. 574-586; duplicated 1953 as U. S. Geological Survey Ground Water Note 8, 1940.

KNUDBY, C.; CARRERA, J. On the Use of Apparent Hydraulic Diffusivity as an Indicator of Connectivity. Journal of Hydrology, n. 329, p. 377-389, 2006.

LI, H.; JIAO, J.J. Tide-induced groundwater fluctuation in a coastal leaky confined aquifer system extending under the sea. Water Resources Research, v. 37, n. 5, p. 11651171, 2001.

LUDVIGSON, J.E.; HJERNE, C. Detailed analysis of selected hydraulic interference tests and review of new test analysis methods. Geosigma AB. Swedish Nuclear Fuel and Waste Management Co, Stockholm, 2014.

MERRITT, M.L. Estimating Hydraulics Properties of the Floridan Aquifer System by Analysis of Earth-Tide, OceanTide, and Barometric Effects, Collier and Hendry Counties, Florida. U.S. Geological Survey, Water Resources Investigations Report 03-4267, 2004. 
OLIVEIRA, L.T. Aspectos Hidrogeológicos da Região Costeira Norte de Pernambuco - Paulista a Goiana. Dissertação (Mestrado) - Universidade Federal de Pernambuco, 2003.

PLANAT/CAERN. Captação de Baraúna, poço tubular no 2 CPRM-04-MO-15-RN. Relatório de conclusão. Relatório inédito, 29 p, 1979.

SÁNCHEZ-ÚBEDA, J.P.; CALVACHE, M.L.; DUQUE, C.; LÓPEZ-CHICANO, M. Estimation of hydraulic diffusivity using tidal-extracted oscillations from groundwater head af fected by tide. 24th Salt Water Intrusion Meeting and the 4th Asia-Pacific Coastal Aquifer Management Meeting, Cairns, Australia, 2016.

SILVA, M. Hidrogeologia do Algarve Oriental. Tese (Doutorado em Geologia) - Departamento de Geologia da FCUL, 1984. 260 p, 1984
STALLMAN, R.W.; PAPADOPULOS, S. Measurement of Hydraulic Diffusivity of Wedge-Shaped Aquifers Drained by Streams. Geological Survey Professional Paper, 514, 1966.

THEIS, C.V. The relation between the lowering of the piezometric surface and the rate and duration of discharge of a well using ground-water storage. Transactions American Geophysical Union, 16th Ann. Meeting, part 2, 1935.

WEN, J.C.; LIN, H.R.; JIM YEH, T.C.; WANG, Y.L.; LIN, K.L.; HUANG, S.Y. Hydraulic Tomography for Estimating the Diffusivity of Heterogeneous Aquifers Based on Groundwater Response to Tidal Fluctuation in an Artificial Island in Taiwan. Geofluids, v. 2018, Article ID 6046258, 15 pages, 2018. 